\title{
Dilation of the Duodenal Limb after Resection of a Huge Jejunal Gastrointestinal Stromal Tumor
}

\author{
Gwang Ha Kim ${ }^{1,2}$, Dae Hwan Kim ${ }^{2,3}$ \\ Department of Internal Medicine, Pusan National University School of Medicine ${ }^{1}$, Biomedical Research Institute, Pusan National University \\ Hospital $^{2}$, Department of Surgery, Pusan National University School of Medicine ${ }^{3}$, Busan, Korea
}

Question: A 70-year-old woman presented with right upper quadrant pain and vomiting. Six months before, she had underwent segmental resection of a huge $13 \mathrm{~cm}^{-}$ sized gastrointestinal stromal tumor in the proximal jejunum and gastrojejunostomy (Fig. 1A). On abdominal CT at visit, marked dilation of the excluded duodenal limb (from the gastric pylorus to the transected distal duodenum) was observed (Fig. 1B).

What is the appropriate treatment?

Answer: Because the duodenal limb dilation was thought to be caused by closed loop formation secondary to the intact pyloric function, endoscopic pyloromyotomy was planned. After submucosal injection of a saline solution mixed with diluted epinephrine $(0.025 \mathrm{mg} / \mathrm{mL})$ and indigo carmine, a mucosal incision was made in the posterior wall of the gastric antrum, $5 \mathrm{~cm}$ proximal to the pylorus (Fig. 2A). Then, a submucosal tunnel was created from the mucosal entry to approximately $1 \mathrm{~cm}$ over the pylorus (Fig. 2B). Full-thickness myotomy was performed from $2 \mathrm{~cm}$ proximal to the pylorus to $1 \mathrm{~cm}$ beyond the pylorus (Fig. 2C). Thereafter, the mucosal entry was closed with metal clips and a detachable snare (Fig. 2D). The next day her symptoms improved and then discharged 3 days later. At 25 months follow-up, no recurrence of symptoms was noted.

As one of the submucosal endoscopies, peroral endo-

Received: May 19, 2019 Revised: June 11, 2019 Accepted: June 12, 2019

Corresponding author: Gwang Ha Kim

Department of Internal Medicine, Pusan National University School of Medicine, Biomedical Research Institute, Pusan National University Hospital, 179 Gudeok-ro, Seo-gu, Busan 49241, Korea

Tel: +82-51-240-7869, Fax: +82-51-244-8180, E-mail: doc0224@pusan.ac.kr

Copyright $\odot 2019$ Korean College of Helicobacter and Upper Gastrointestinal Research

@ The Korean Journal of Helicobacter and Upper Gastrointestinal Research is an Open-Access Journal. All articles are distributed under the terms of the Creative Commons Attribution Non-Commercial License (http:// creativecommons.org/licenses/by-nc/4.0) which permits unrestricted non-commercial use, distribution, and reproduction in any medium, provided the original work is properly cited. scopic myotomy is known as one of the safe and efficient treatment modalities for esophageal achalasia with a higher clinical outcomes success rate over $90 \% .{ }^{1}$ This technique can be also adopted to other functional gastrointestinal disorders, including gastroparesis. In recent decade, gastric peroral endoscopic pyloromyotomy has been widely explored as a py-
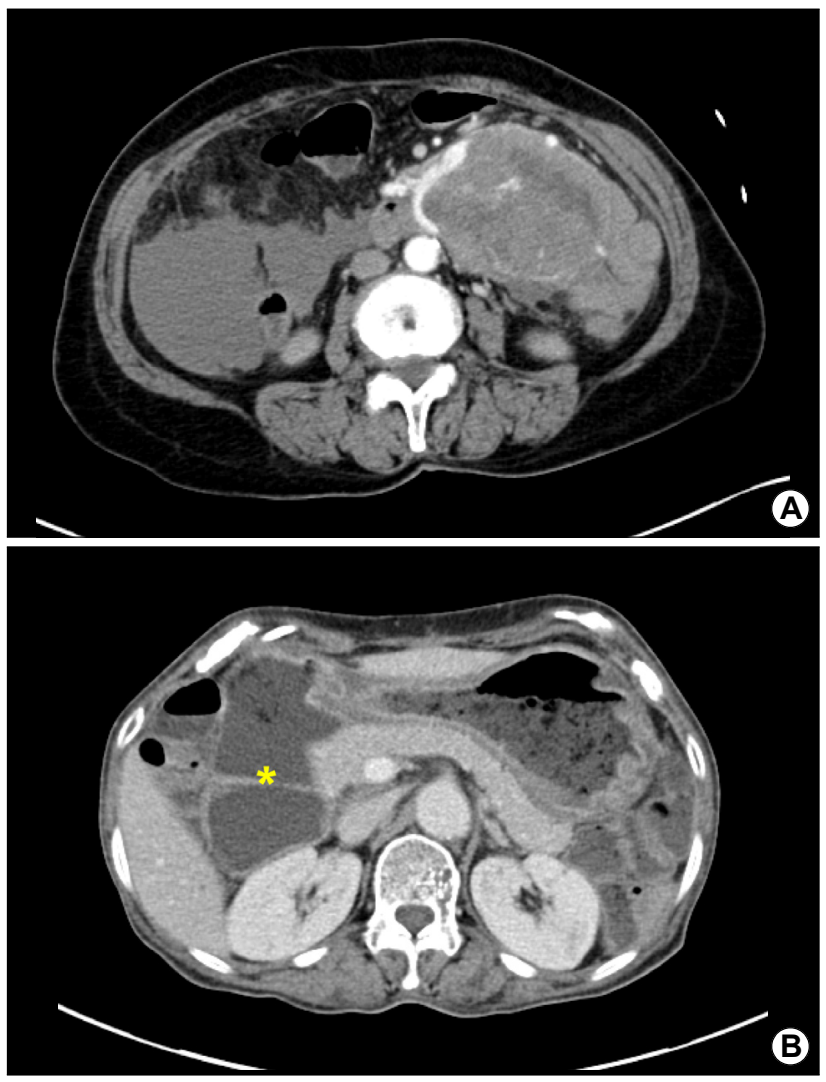

Fig. 1. (A) Abdominal computed tomography (CT) at the time of segmental resection and gastrojejunostomy (6 months prior) showing a huge 13-cm-sized gastrointestinal stromal tumor in the proximal jejunum. (B) Abdominal CT at hospital visit revealing marked dilation of the excluded duodenal limb (asterisk, from the gastric pylorus to the transected distal duodenum). 

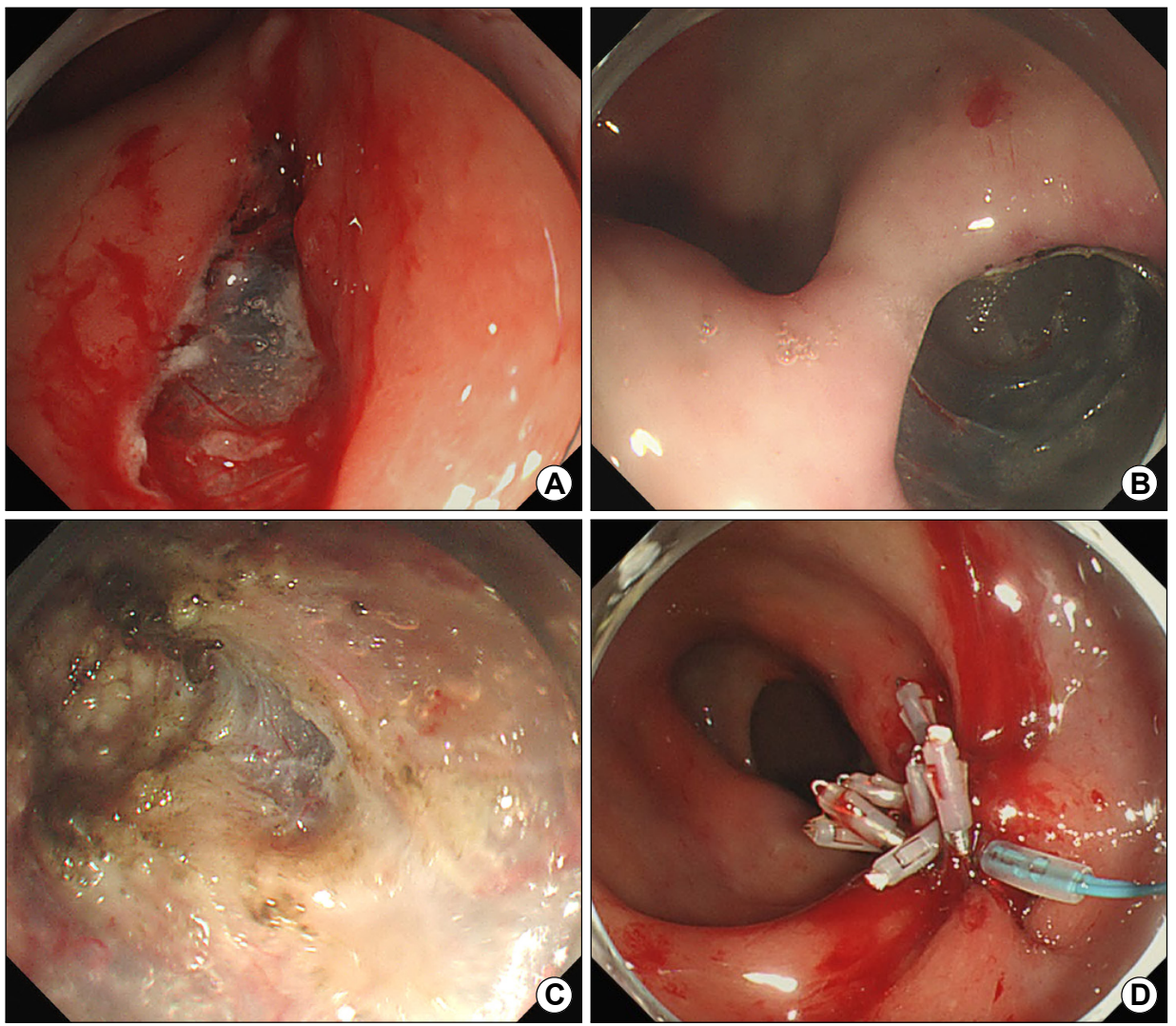

Fig. 2. (A) Creation of a mucosal incision in the posterior wall of the gastric antrum $5 \mathrm{~cm}$ proximal to the pylorus. (B) Creation of a submucosal tunnel from the mucosal entry to approximately $1 \mathrm{~cm}$ beyond the pylorus. (C) Performance of full-thickness myotomy from $2 \mathrm{~cm}$ proximal to the pylorus to $1 \mathrm{~cm}$ beyond the pylorus. (D) Closing of the mucosal entry with metal clips and a detachable snare.

lorus-directed therapy for gastroparesis. ${ }^{2}$

In the excluded duodenal limb dilation caused by the intact pylorus, mechanical or functional disruption of the pyloric muscle can be one of treatment options. Disruption of the pyloric muscle is possible by surgical pyloroplasty, transpyloric stenting, or intrapyloric botulinum injection. ${ }^{3}$ Similarly to esophageal achalasia, endoscopic pyloromyotomy can be a substitute to the surgical pyloroplasty in the excluded duodenal limb dilation caused by the intact pylorus.

\section{CONFLICT OF INTEREST}

No potential conflict of interest relevant to this article was reported.

\section{ORCID}

Gwang Ha Kim (D) https://orcid.org/0000-0001-9721-5734

Dae Hwan Kim (D) https://orcid.org/0000-0001-7450-797X

\section{REFERENCES}

1. Awaiz A, Yunus RM, Khan S, Memon B, Memon MA. Systematic review and meta-analysis of perioperative outcomes of peroral endoscopic myotomy (POEM) and laparoscopic heller myotomy (LHM) for achalasia. Surg Laparosc Endosc Percutan Tech 2017;27:123-131.

2. Mekaroonkamol P, Shah R, Cai Q. Outcomes of per oral endoscopic pyloromyotomy in gastroparesis worldwide. World J Gastroenterol 2019;25:909-922.

3. Clarke JO, Snape WJ Jr. Pyloric sphincter therapy: botulinum toxin, stents, and pyloromyotomy. Gastroenterol Clin North Am 2015;44:127-136. 